\title{
First-principles study of the interaction between helium and the defects in tantalum
}

\author{
Wen Yin ${ }^{\text {a, }}{ }^{, b}$, Xuejun Jia ${ }^{\text {a, b }}$, Quanzhi Yu a, b, Tianjiao Liang a, b \\ ${ }^{a}$ Spallation Neutron Source Engineering Center for Target and Instruments, Beijing National Laboratory for Condensed Matter Physics, Institute of Physics, Chinese Academy of \\ Science, 100190, China \\ ${ }^{\mathrm{b}}$ Dongguan Branch, Institute of High Energy Physics, CAS, Beijing 100049, China
}

\begin{tabular}{ll}
\hline A R T I C L E I N F O & A B S T R A C T \\
\hline $\begin{array}{l}\text { Article history: } \\
\text { Received } \\
\text { Avcepted }\end{array}$ & $\begin{array}{l}\text { As a low power spallation target or target cladding material, tantalum has almost the same defects productions as } \\
\text { tungsten under irradiation. In this paper, the detailed analysis of helium behavior in tantalum has been conducted } \\
\text { based on density functional theory. The formation energy of tetrahedral interstitial configuration of helium is } 0.3 \mathrm{eV} \\
\text { lower than that of octahedral interstitial configuration, which means the tetrahedral interstitial configuration of } \\
\text { helium is more stable than the octahedral configuration. Tetrahedral helium atoms in tantalum are predicted to have a } \\
\text { Helium behaviors } \\
\begin{array}{l}\text { Density functional theory } \\
\text { Tantalum }\end{array}\end{array} \quad \begin{array}{l}\text { very low migration, about } 0.09 \mathrm{e} V \text {. A large binding energy between He and vacancy } 2.03 \mathrm{eV} \text { indicates that He atom is } \\
\text { easily trapped by vacancy, and a tetrahedral interstitial helium atom need overcome about } 0.19 \mathrm{e} \text { energy barriers } \\
\text { when it directly jumps into the vacancy. Finally, the helium behavior in tantalum is compared with that in tungsten. It } \\
\text { is obvious that the binding energy in tantalum is significantly different from those in tungsten. The interaction } \\
\text { between two helium atoms in tantalum shows repulsive or weak repulsive other than attractive in tungsten, which } \\
\text { suggests that helium atoms are easy to move other than to be a cluster in tantalum. }\end{array}$ \\
\hline
\end{tabular}

\section{Introduction}

Due to high neutron production and high melting point, tungsten is being considered for use as a target material in many spallation neutron sources such as CSNS [1], ISIS [2] and KENS [3]. Another refractory metal tantalum also can yield many neutrons under spallation conditions that are comparable to tungsten. Although tantalum has much higher afterheat and activity than tungsten when it is bombarded by high-energy protons or neutrons, it surpasses tungsten in ductility, workability and weld ability $[4,5]$. Furthermore, it forms highly stable anodic films on its surface, which increases tantalum chemical stability largely. Thus tantalum is a candidate target or target cladding material for low power spallation neutron sources $[6,7]$.

The spallation neutron sources are usually propelled by protons whose energies range from $800 \mathrm{MeV}$ to $3 \mathrm{GeV}$. At such high energies, the spallation neutrons lead to the production of large amounts of helium, hydrogen and new heavier species in forms of transmutation products. The tungsten and tantalum have similar helium production cross sections under spallation reaction [8].These large amounts of helium, hydrogen and heavier transmutation products may exacerbate the displacement radiation damage [9]. Recent experiments led to the conclusion that helium caused a larger increase in yield stress than that due to displacement damage and any irradiation-induced precipitation hardening. An increase in the ductile-brittle transition temperature (DBTT) had been found experimentally due to helium and there is a linear relationship between $\triangle \mathrm{DBTT}$ and helium concentration for steel irradiated over the range 2.5-18.4dpa and 85-1530appm $\mathrm{He}[10,11]$

\footnotetext{
* Corresponding author. The Institute of Physics, Chinese Academy of Sciences, P.O.Box 603, Beijing 100190, China.

E-mail address: wenyin@aphy.iphy.ac.cn (Yin Wen)
}

Much attention has been paid in helium production and much is still devoted to the study of the behavior of helium in metals such as Helium migration, diffusion, trapping, dissolution as well as their relation with the change in mechanical properties [12-15].

Because of the highly important role of helium in irradiated metals, especially for spallation neutron source and fusion materials, there are extensive works on tungsten, iron or zirconium alloys [12-18]. Thus this work aims at investigating the formation energy and migration behavior of helium in tantalum. Firstly, the favorite positions of the spallation defects in tantalum are investigated by determining the formation energy of these defects. Then the interaction between $\mathrm{He}$ and other spallation defects is considered, and there exists a large binding energy between He and vacancy. Finally the heliumhelium binding energies in tantalum is compared with those in tungsten. We surmise due to the sharp drop of the $5 d$ projected density of states around Fermi level, the interactions between helium atoms in tantalum are obviously different from those in tungsten.

\section{Defect formation energy in tantalum}

Although helium, hydrogen and self-interstitial atoms (SIA) are relatively rare in metals compared with vacancies under normal condition, they are plentiful and of prime importance in high-energy radiation environment. For example, under spallation helium can be produced in target by $(n, \alpha)$ transmutation reactions and the ratio of helium production to displacement (He/dpa ratio) is much higher than fission neutrons or even fusion neutrons [19]. For tantalum, helium forms by a two-step reaction of ${ }^{181} \mathrm{Ta}$. Step one, ${ }^{181} \mathrm{Ta}$ transmutes to ${ }^{182} \mathrm{Ta}$ with thermal neutrons by (n, gamma) radiative capture; step two, helium forms in ${ }^{182} \mathrm{Ta}$ with fast neutrons by (n, a) reaction. We firstly calculate the helium, hydrogen, self-interstitial atoms (SIA), and vacancy formation energies for tantalum.

In body centered cubic (bcc) metal all substitution positions are equivalent and there are two interstitial positions: the octahedral and the tetrahedral positions. The location of helium in metals influences its migration; the more 
favored interstitial position in tantalum is calculated first. The following calculation had been performed on the basis of the frozen-core projected augmented wave (PAW) method within the Vienna ab initio Simulation Package VASP [20], where the exchange and correlation effects are described by the density functional theory (DFT) within the generalized gradient approximation (GGA) of Perdew and Wang [21]. For the plane-wave set, a cut off energy of $350 \mathrm{eV}$ is used [12]. Except when otherwise mentioned, all the results presented below are obtained using 128 atoms and a $3 \times 3 \times 3$ k-points grid. The super-cell contains 128 atoms are fully relaxed until the HellmannFeynman forces become less then $0.02 \mathrm{eV} / \AA$. The equilibrium lattice parameters obtained for tantalum is $3.3094 A$, which is a little higher than the experimental data $3.3013 \AA$ [22]

The helium, self-interstitial atoms and vacancy formation energies in tantalum were calculated by

$$
\begin{aligned}
& E_{H e}^{f}=E(N T a+H e)-E(N)-E\left(H e_{i s o l a t e d}\right), \\
& E_{I}^{f}=E_{t o t a l}(N+1)-\frac{N+1}{N} E_{t o t}(N), \\
& E_{V}^{f}=E_{t o t a l}(N-1)-\frac{N-1}{N} E_{t o t}(N) .
\end{aligned}
$$

Where $E\left(N T_{a}+H e\right)$ is is the energy of the super-cell containing $\mathrm{N}$ Ta atoms and $1 \mathrm{He}$ atom, and $E(N)$ is the reference energy of Ta in a perfect bcc structure, the $E\left(H_{\text {isolated }}\right)$ is an isolated He atom, $E_{\text {toal }}(N+1), E_{\text {total }}(N-1)$ are the total energy of the supercell with a self-interstitial and with a vacancy, respectively.

Table 1 gives the formation energies of the defects in tantalum. As a consequence the predicted favored interstitial site of helium in tantalum is tetrahedral site and the energy difference between octahedral and tetrahedral is $0.31 \mathrm{eV}$, as shown in table 1 . The results shows that the formation energy of hydrogen in tantalum is negative, which means tantalum can absorb hydrogen atoms. The $<111>$ dumbbell is the most stable configuration in tantalum. In this paper, we investigate the kinetics of helium in tantalum which is very important for the understanding the He migration, bubble nucleation or dissociation.

\section{The interaction between helium and the other defects in tantalum}

The Fundamental properties of helium in metals including the equilibrium structure and the migration barrier are usually inferred from direct measurements on irradiated samples. There exist some experiments on investigating the mechanical properties after spallation irradiation; however the migration, clustering or dissolution of helium at the high defect densities in tantalum may obscure the interpretation of experimental data.

Firstly He migration is examined. Here the climbing image nudged elastic band (CINEB) method is employed to find the minimum energy path (MEP) of the $\mathrm{He}$ atom migration when the initial and final configurations are known. Migration energy barrier is defined as the energy difference between an initial configuration and the saddle point.

A tetrahedral He will migrate between two equivalent sites without passing through an octahedral site. Very low migration energy, $0.09 \mathrm{eV}$, is found for $\mathrm{He}$ in tantalum, as shown in figure 1. Such a low migration energy means that the migration of interstitial $\mathrm{He}$ is almost athermal.

Since it is easy that $\mathrm{He}$ atom mobiles in tantalum, it will encounter other defects such as another He atom, Hydrogen atom, vacancy or SIA, even in very low He concentration. We now turn to the interaction between the He atom and the other defects. Here we assumed that the defects are located their most stable positions. The binding energy for helium-helium atoms is obtained as

$$
\boldsymbol{E}^{b}\left(\mathrm{He}_{1}, \mathrm{He}_{2}\right)=\boldsymbol{E}^{f}\left(\mathrm{He}_{1}\right)+\mathrm{E}^{f}\left(\mathrm{He}_{2}\right)-\mathrm{E}^{f}\left(\mathrm{He}_{1}, \mathrm{He}_{2}\right),
$$

and the binding energy for helium and other defects is obtained as

$$
\begin{aligned}
& \boldsymbol{E}^{b}(\mathrm{He}, \mathrm{Vac} .)=\boldsymbol{E}^{f}(\mathrm{He})+\boldsymbol{E}^{f}\left(\mathrm{Vac}_{.}\right)-\boldsymbol{E}^{f}(\mathrm{He}, \mathrm{Vac} .), \\
& \boldsymbol{E}^{b}(\mathrm{He}, \boldsymbol{H})=\boldsymbol{E}^{f}(\mathrm{He})+\boldsymbol{E}^{f}(\boldsymbol{H})-\boldsymbol{E}^{f}(\mathrm{He}, \boldsymbol{H}),
\end{aligned}
$$

$E^{b}(H e, S I A)=E^{f}(H e)+E^{f}(S I A)-E^{f}(H e, S I A)$, (7) where $E^{f}(\mathrm{He}), E^{f}(\mathrm{Vac}),. E^{f}(H), E^{f}(\mathrm{SLA})$ are the formation energies for a tetrahedral helium atom, a vacancy, a tetrahedral hydrogen atom, and SIA, respectively. $E^{f}(\mathrm{He} / \mathrm{Vac} . / \mathrm{H} / \mathrm{SLA})$ is the formation energy that super-cell contains a helium atom and a nearest vacancy, or a tetrahedral hydrogen atom, or SIA atoms with the $<111>$ configuration. Table 2 shows that the interactions in tantalum between two helium atoms, between a helium atom and a hydrogen atom, and between a helium atom and SIA atoms are repulsive. But the interaction between a helium atom and a vacancy is attractive. Furthermore, the attraction between a vacancy and a helium atom is so strong that the helium atom will be trapped by the vacancy and we do find the helium atom relaxes a lot towards to the vacancy position. It thus explains why small diffusion constants of helium in tantalum are obtained in the previous experiments, in which the authors thought the small value hinted to strong helium trapping by vacancies and $\mathrm{He}-\mathrm{V}$ cluster formation [23].

Since there exist some interactions between helium and the other defects, the diffusion of a tetrahedral helium atom is considered, in the presence of another a hydrogen atom, or a vacancy, or a SIA. The migration mechanism is the diffusion of $\mathrm{He}$ from one tetrahedral interstitial site (TIS) into the nearest neighbor TIS, in the presence of a hydrogen atom, as shown in figure 2.

Actually it is easy to deduce that the repulsive energy between $\mathrm{He}$ and $\mathrm{H}$ atoms pushes $\mathrm{He}$ atom away from the $\mathrm{H}$ atom, which can be seen in Fig.2. The minimum energy path of the He atom shows it will jump into the nearest TIS without any energy barrier.

Then the diffusion of $\mathrm{He}$ from one TIS into the nearest vacancy is considered. Figure 3 shows the energy barrier can be obtained as about $0.19 \mathrm{eV}$, which must be overcome in the direct diffusion path of He from the TIS into the nearest vacancy.

From the above discussion, we find that the repulsive interaction between $\mathrm{He}$ atom and $\mathrm{H}$ atom, pushes the $\mathrm{He}$ atom to escape from the defect. For a tetrahedral $\mathrm{He}$ atom, the nearest vacancy is a trap site and this atom need overcome about $0.19 \mathrm{eV}$ energy barriers when it directly jumps into the vacancy.

\section{Comparison of the He-He interaction between tantalum and tungsten}

Since tantalum and tungsten are the most commonly used spallation target materials and they have almost the same He production when they are bombarded by high-energy particle, it is interesting to compare the He behavior in tantalum with that in tungsten. Previous work had shown that there was a large binding energy between $\mathrm{He}-\mathrm{He}$ atoms in tungsten, which indicates that $\mathrm{He}$ atoms can be trapped by other He atoms to form a He cluster [12]. We then consider the interaction between $\mathrm{He}$ atoms that are in the different tetrahedral interstitial sites (see figure 4).

The results are summarized in table 3 as well as the previous results [12]. For comparison, here we also give the binding energies in tungsten. It is obviously that the binding energies in tantalum are significantly different from those in tungsten. The interaction between the two $\mathrm{He}$ atoms in tantalum shows repulsively and weak repulsively other than attractively in tungsten. The repulsive or weak repulsive interaction indicates the two He atoms are more difficult to become a cluster in tantalum than in tungsten and the He cluster is much less stable in tantalum than in tungsten. It is worth mentioning that the presence of the interstitial He atoms cause a noticeable lattice distortion both in tantalum and tungsten. For the first nearest neighbor arrangement, the Ta atom located in the nearest neighbor of $\mathrm{He}$, had displaced $0.33 \AA$ from its original position; the $\mathrm{W}$ atom, has moved about $0.35 \AA$. Moreover, when we exploring the distance of two He atoms, we note the He atoms in tungsten tend to reduce the distance between them (not shown).

To show this discrepancy between these two heavy metals, similar analysis had been done between three and four He atoms clusters. The binding energies between three $\mathrm{He}$ atoms in tantalum and tungsten are $-0.059 \mathrm{eV}$ and $2.27 \mathrm{eV}$, respectively. Those between four He atoms in tantalum and tungsten are $0.57 \mathrm{eV}$ and $3.40 \mathrm{eV}$, respectively. The results show the same trend for tantalum and tungsten, which are weak repulsive interactions between two He atoms in tantalum and strong attractive interactions in tungsten.

\section{Discussion}


From the above calculations, it is energetically easy for He moving both in tungsten and in tantalum. Furthermore, the different He-He binding energies in tantalum and tungsten indicate that clustering of two He atoms in tungsten is very probable, but it is obviously not the case in tantalum. Since these two heavy metals have similar crystal structures, the distinct discrepancy between these two heavy metals may be understood in terms of the electronic density of states (DOS). The valence configurations are $4 \mathrm{f}^{14} 5 \mathrm{~d}^{4} 6 \mathrm{~s}^{2}$ and $4 \mathrm{f}^{14} 5 \mathrm{~d}^{3} 6 \mathrm{~s}^{2}$ for tungsten and tantalum, respectively. Calculation of the $5 d$ site-projected DOS for pure tungsten and tantalum after relaxation reveals that in pure tantalum there exists a sharp decrease of the DOS just around the Fermi level and the Fermi level lies at the maximum of the sharp peak; however, this structure is totally absent in tungsten (see Fig.5). The strong variation of the DOS just around Fermi level in tantalum is considered to increase the electronic entropy of tantalum. Here we guess it probably induces the different binding energies of the $\mathrm{He}$ atoms between tantalum and tungsten.

Then we have calculated the $p d$ site-projected density of states (DOS) of the tetrahedral interstitial $\mathrm{He}$ and its first nearest neighboring Ta atom. In figure 6, the first thing to note is the overall similarity in the shapes of Ta $5 d$ and $\mathrm{He} p$ projected DOS that reflects the strong hybridization of Ta and He atoms. This hybridization suggests there is a strong electronic interaction between Ta and $\mathrm{He}$ atoms. This hybridization also can be found in $\mathrm{He}$ and its first neighboring $\mathrm{W}$ atom as well (not shown). This strong hybridization of parent atom and $\mathrm{He}$ electronic states may explain a repulsive interaction between them, which make parent atom be depart from its original location as we mentioned above. At the same time there still exits a sharp decrease of the DOS around the Fermi level in tantalum as pure tantalum.

\section{Conclusion}

The helium behaviors of helium atoms in tantalum have been systematically calculated with density functional theory. The results of the formation energies of the common spallation defects and the influence of these defects on diffusion of $\mathrm{He}$ atoms are presented. Results show that the He atoms will stay in the tetrahedral site in tantalum. The tetrahedral He atoms will migrate between two equivalent sites, and the migration energy is about $0.09 \mathrm{eV}$. The repulsive energy between $\mathrm{He}$ and $\mathrm{H}$ atoms pushes $\mathrm{He}$ atom away from $\mathrm{H}$ atom without any barrier. A helium atom need overcome about $0.19 \mathrm{eV}$ energy barriers when it directly jumps into the vacancy. The results also shows that the interaction between $\mathrm{He}$ atoms and defects in tantalum is repulsive, but is strong attractive in tungsten and it is concluded that it is much more difficult to form the $\mathrm{He}$ clusters in tantalum other than in tungsten, although these two refractory metals are similar in He productions under spallation reaction,

\section{Acknowledgment}

This work was supported by the National Science Foundation of China (Grant No.51371195 and 11174358).

\section{References}

[1] W.Yin, Q.Z.Yu, Y.L.Lu, S.L.Wang, J.F.Tong, T.J.Liang, J. Nucl. Mater. 431 (2012) 39

[2] S.M.Bennington, S.I.Campbell, T.A.Broome, D.J.Picton and T.D.Beynon, Journal of Neutron Research 11 (2003) 93

[3] M.Kawai, K.Kikuchi, H.Kurishita, J.Li, M.Furusaka, J. Nucl. Mater. 296 (2001) 312

[4] J.Chen, H.Ullmaier, T.Flobdorf, et.al., J. Nucl. Mater. 298 (2001) 248

[5] J.Chen, P.Jung, M.Rodig, H.Ullmaier, G.S.Bauer, J. Nucl. Mater. 343 (2005) 227

[6] T hak Sang Byun, Stuart A. Maloy, J. Nucl. Mater.377 (2008) 72

[7] Q.Z.Yu, T.J.Liang, and W.Yin, Radiat. Prot. Dosim.136 (2009)216

[8] R.A.Lillie, F.X.Gallmeier, Hilo2k: A New Hilo Library to $2 \mathrm{GeV}$, Oak Ridge National Laboratory, 2003

[9] W ei Lu, Monroe S. Wechsler, Phillip D. Ferguson, and Eric J. Pitcher, Journal of ASTM International, Vol.3, No.7, Paper ID JAI13467 (2006)

[10] R.L.Klueh, N.Hashimoto, M.A.Sokolov, P.J.Maziasz, K.Shiba, S.Jitsukawa, J. Nucl. Mater. 357 (2006) 169

[11] Y.Dai, X.Jia, R.Thermer, F.Groeschel, Irradiation Effects in Martensitic Steel Under Neutron and Proton Mixed Spectrum, TM-34-04-08, Paul Scherrer Institute, October 15, 2004

[12] C harlotte S. Becquart and Christophe Domain, Physical Review Lett. 97 (2006)196402

[13] H. Trinkaus and B. N. Singh, J. Nucl. Mater.323 (2003) 229

[14] E nrique Martinez and Chu-Chun Fu, Physical Review B 84 (2011)014203

[15] T atiana Seletskaia, Yuri Osetsky, R.Z.Stoller and G.M.Stocks, Physical Review Lett. 94 (2005) 046403

[16] H. Amara, C. C. Fu, F. Soisson and P. Maugis, Physical Review B 81 (2010) 17410

[17] Peng Zhang, Yong Lu, Chao-Hui He, Ping Zhang, J. Nucl. Mater. 418 (2011) 143

[18] R. Vassen, H. Trinkaus and P. Jung, Physical Review B 44 (1991) 4206

[19] W. Lu and M.S.Wechsler, J. Nucl. Mater. 361 (2007) 282

[20] G. Kresse and D.Joubert, Phys. Rev. B 47 (1993) 558; 49 (1994) 14251

[21] J.P.Perdew, J.A.Chevary, S.H.Vosko, K.A.Jackson, M.R.Pederson and C.Fiolhais, Phys. ReV. B 46 (1992) 6671

[22] W.R.Morcom, W.L.Worrell, H.G.Sell, and H.I.Kaplan, Metallurgical transactions 5 (1974) 155

[23] F.Zielinski, J.M.Costantini, J.Haussy, and F.Durbin, Journal of Nuclear Materials 312(2003)141

\section{Figure caption}

Figure 1 initial (left) and final (right) configurations of He. Black and gray spheres represent helium, and tantalum atoms, respectively.

Figure 2 initial (left) and final (right) configurations of He. Black, gray and red spheres represent helium, tantalum and hydrogen atoms, respectively.

Figure 3 initial (left) and final (right) configurations of He. Black, gray spheres and dash circle present helium, tantalum atoms and vacancy, respectively.

Figure4 the positions of the tetrahedral He-He atoms. Black and gray spheres represent helium and tantalum or tungsten atoms, respectively. The distances of He-He atoms are as follows (a) $0.354 a$; (b) $0.5 a$; (c) $0.612 a$; (d) $0.707 a$; (e) $0.791 a$; (f) $a$. Here $a$ is the lattice parameters.

Figure 5 comparisons of the $5 d$ site-projected DOS between tantalum and tungsten. The Fermi energy level is set to $0 \mathrm{eV}$.

Figure $65 d$ site-projected DOS of tantalum atom. The $p$ site-projected DOS of the corresponding helium atoms are shown in the inset of the figures. The Fermi energy level is set to $0 \mathrm{eV}$. 
Table 1

Formation energies (in eV) of He, H, vacancy and self-interstitial defects with the different configurations in tantalum. The crowdion SIA (not represented) is nearly degenerate with the $<111>$ configuration.

\begin{tabular}{|c|c|c|c|c|c|c|}
\hline Configuration & Octahedral & Tetrahedral & substitution & $<111>$ & $\langle<110>$ & $\mid<100>$ \\
\hline Helium & 3.65 & 3.34 & 4.58 & & & \\
\hline Hydrogen & -3.70 & -3.37 & -0.08 & & & \\
\hline SIA & 5.49 & 5.30 & & 4.32 & 4.96 & 5.41 \\
\hline Vacancy & & & 3.27 & & & \\
\hline
\end{tabular}

Table 2

The binding energies $(\mathrm{eV})$ and distance variations between helium and the other defects. $a$ is the lattice parameters.

\begin{tabular}{lccc}
\hline \hline & \multicolumn{2}{c}{ The binding energy between helium and the other defects } \\
Helium & Hydrogen & Vacancy & SIA \\
\hline-0.14 & -0.48 & 2.03 & -0.31 \\
& The initial distance between helium and the other defects \\
Helium & Hydrogen & Vacancy & SIA \\
$0.354 \mathrm{a}$ & $0.354 \mathrm{a}$ & $0.559 \mathrm{a}$ & $0.560 \mathrm{a}$ \\
& The final distance between helium and the other defects after relaxation \\
Helium & Hydrogen & Vacancy & SIA \\
$0.464 \mathrm{a}$ & $0.584 \mathrm{a}$ & $0.0110 \mathrm{a}$ & $1.030 \mathrm{a}$ \\
\hline \hline
\end{tabular}

Table 3

The He-He binding energies $(\mathrm{eV})$ in tantalum and tungsten. The calculations were done using 54 atoms and $64 k$ points as well as 128 atoms and $27 k$ points.

The positions refer to figure $5 . *$ : see reference [12]

\begin{tabular}{lcccccc}
\hline \hline & \multicolumn{7}{c}{ The He-He binding energy } \\
He-He Initial distance & $0.354 a$ & $0.5 a$ & $0.612 a$ & $0.707 a$ & $0.791 a$ & $a$ \\
\hline Tantalum/ 54atoms & -0.14 & 0.012 & -0.048 & -0.15 & -0.13 & -0.23 \\
Tantalum/ 128atoms & -0.10 & 0.050 & 0.022 & -0.090 & -0.080 & -0.17 \\
Tungsten/ 54atoms & 0.72 & 0.82 & 1.14 & 0.48 & 1.11 & 0.07 \\
Tungsten/ 54atoms* & 0.68 & 0.87 & 0.98 & 0.29 & 0.96 & -0.06 \\
Tunagsten/ 128atoms & 0.71 & 0.91 & 1.00 & 0.30 & 1.00 & -0.05 \\
Tunagsten/128 atoms* & 0.74 & 0.94 & 1.01 & 0.31 & 1.03 & -0.04 \\
\hline \hline
\end{tabular}




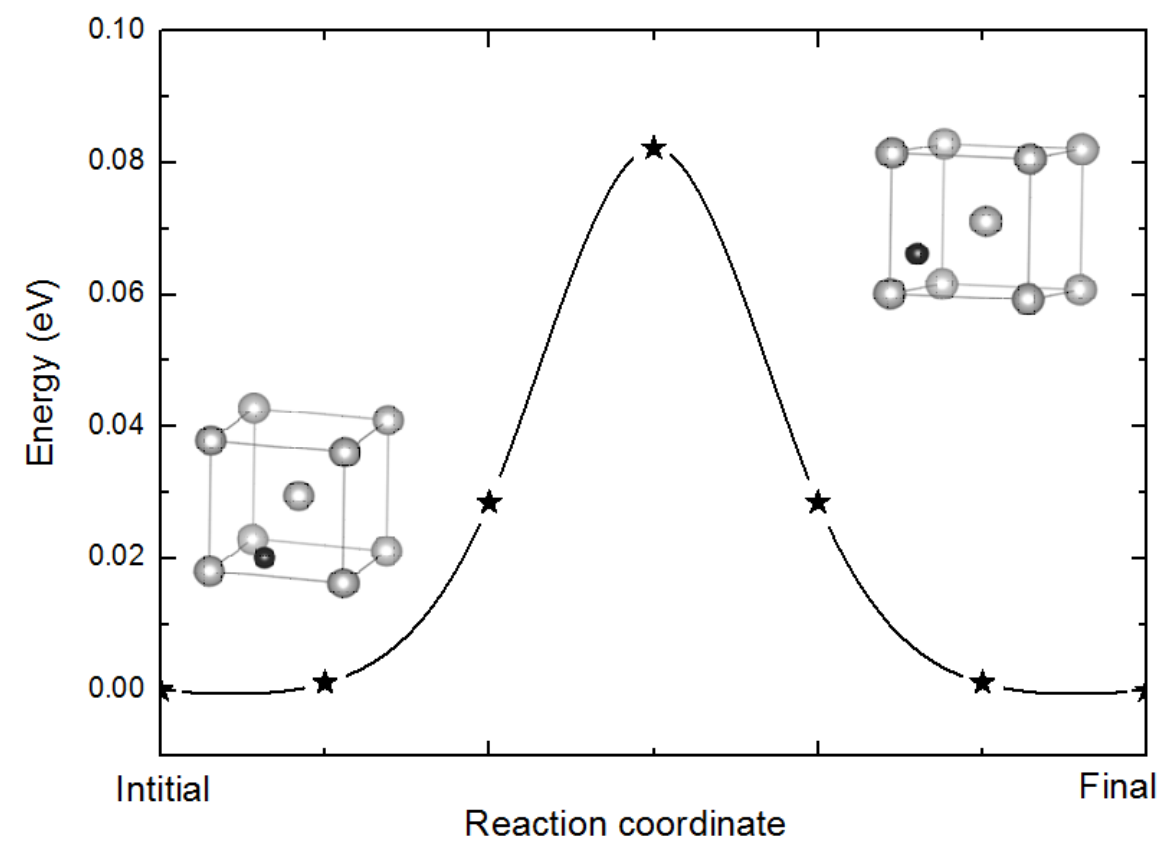




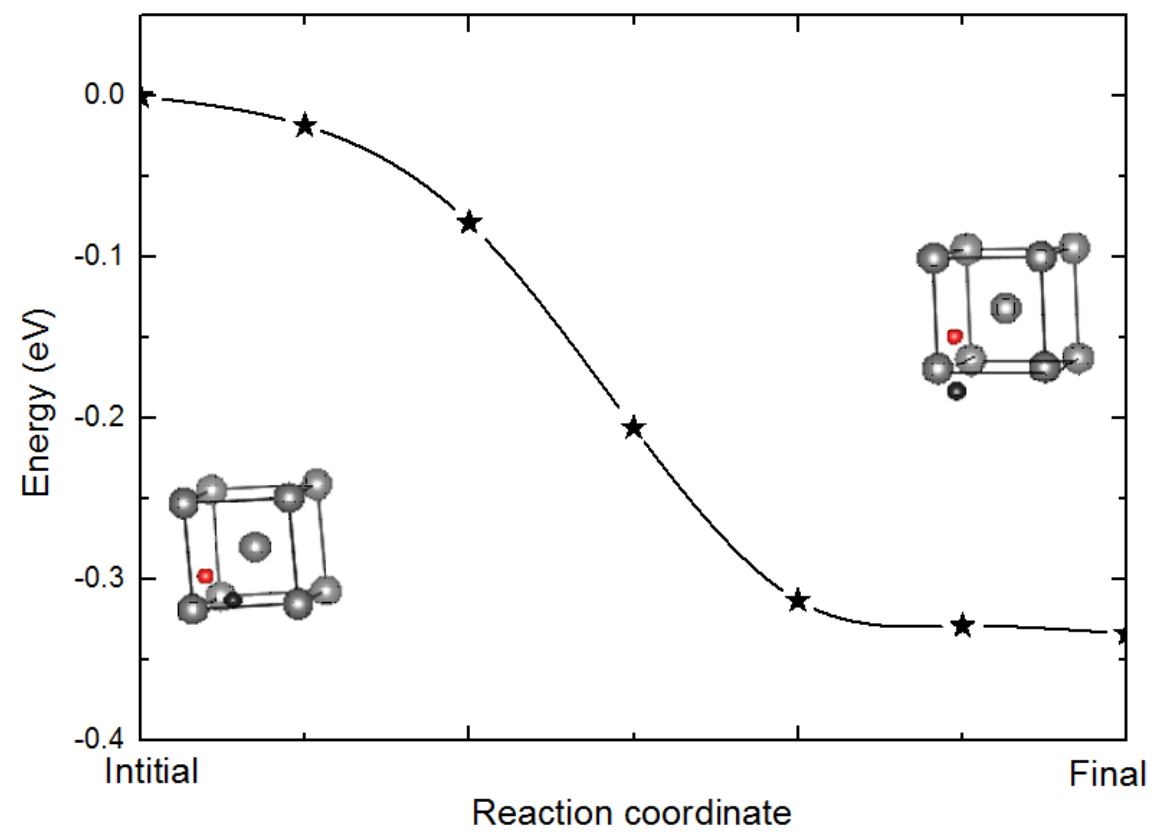




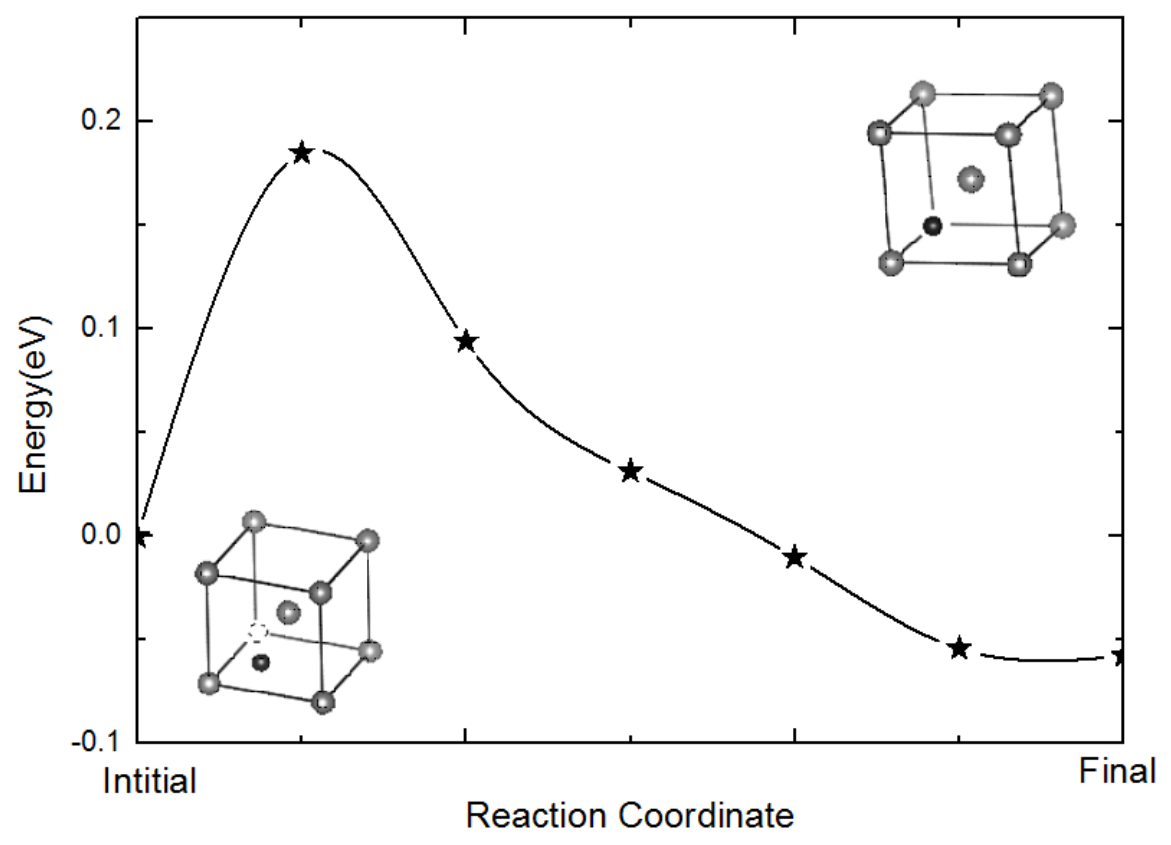




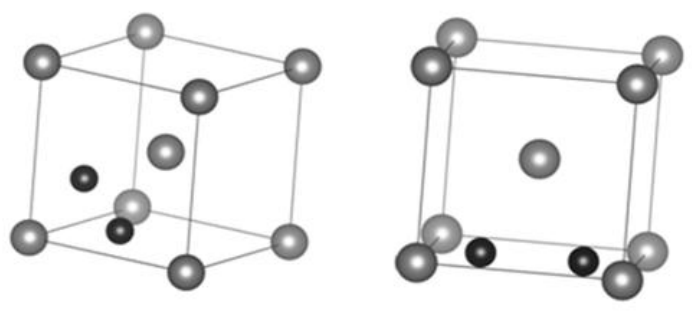

(a)

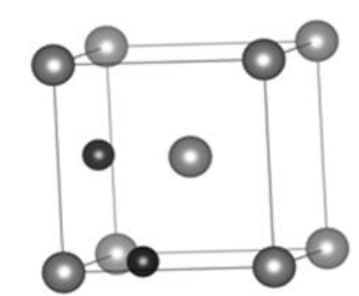

(c)
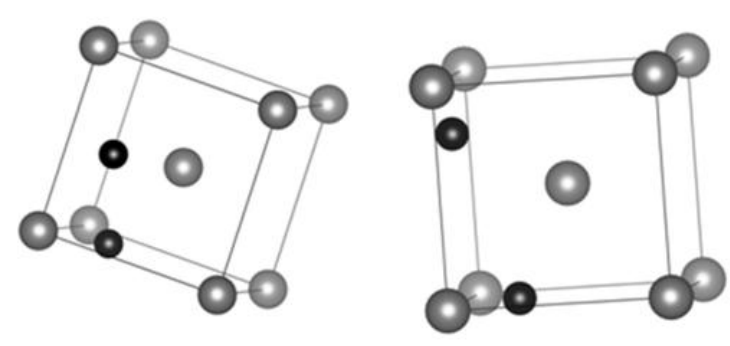

(d)

(e)

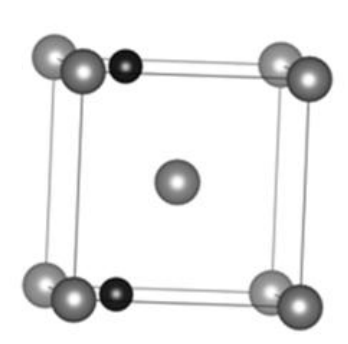

(f) 


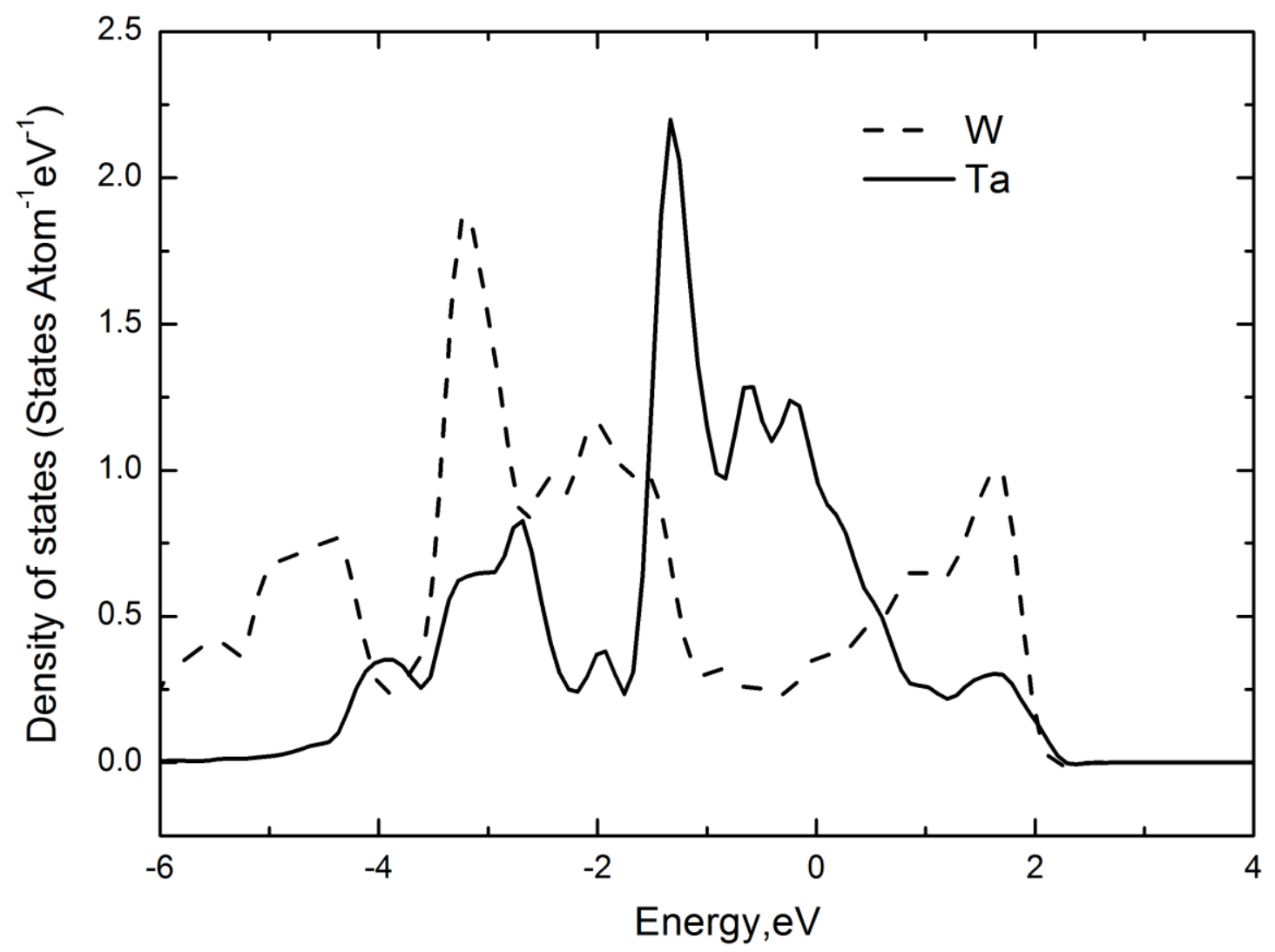




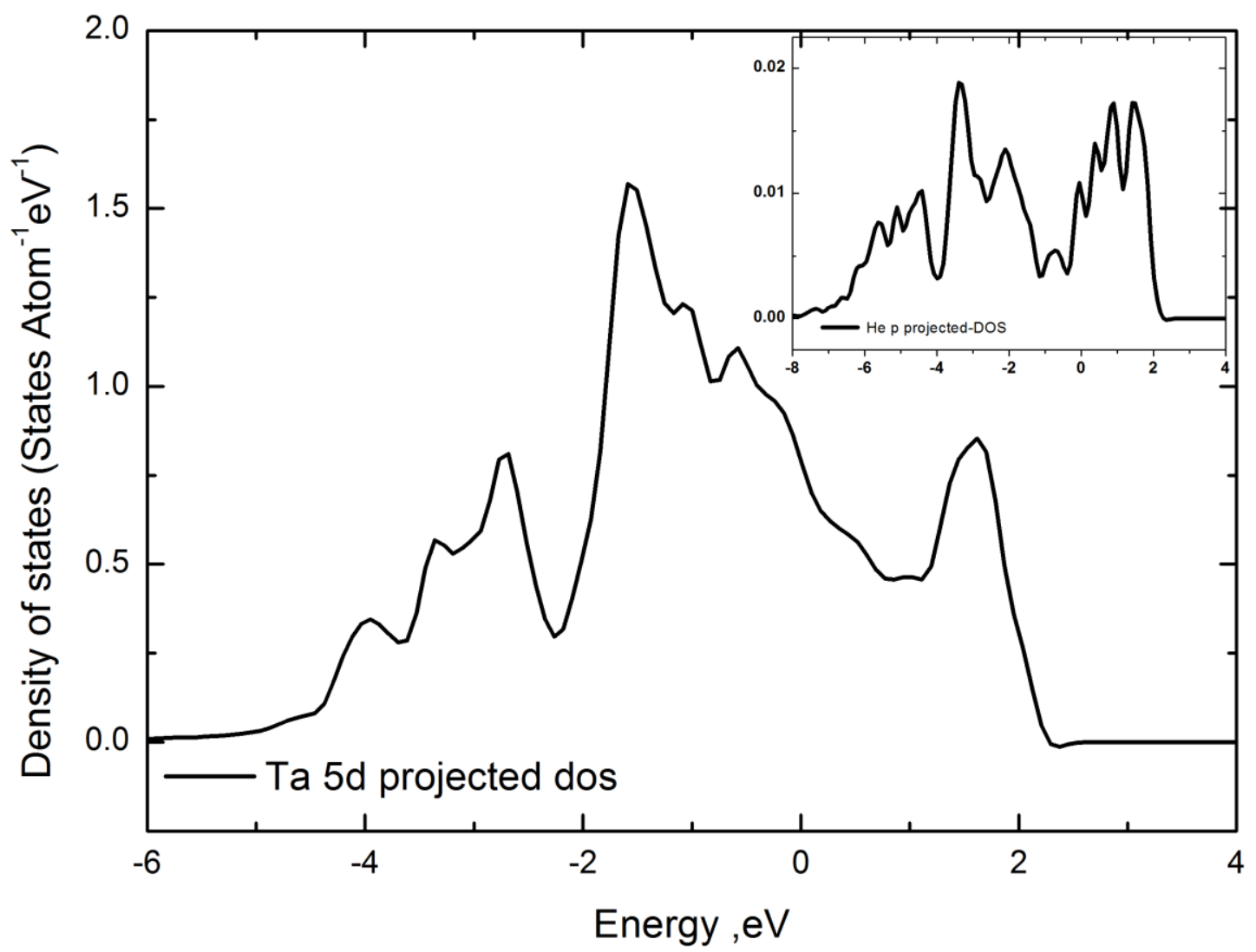

